\title{
Formwandel der Rüstungskontrolle? Rückblick, Bilanz, Ausblick
}

\begin{abstract}
„Was macht eigentlich die Rüstungskontrolle?“ Das mag sich mancher Beobachter fragen, der sie wie eine alte Bekannte vor Zeiten aus den Augen verlor. In den sechziger und siebziger Jahren ließ das Konzept die Wellen hochschlagen: Während radikale Kritiker damals den Ansatz aus der Friedensforschung ausgrenzten oder ihn an ihren äußersten Rand verbannten, rückten Anhänger ihn ins Zentrum der Aufmerksamkeit. Bereits die Übersetzung von arms control löste Kontroversen aus: Einige favorisierten das wortgetreue „Rüstungskontrolle“, andere hielten „kooperative Rüstungssteuerung“ für präziser. Mittlerweile verlässt die Debatte nur noch selten die Expertenzirkel. Erreicht sie doch einmal eine größere Öffentlichkeit, könnte der interessierte Beobachter sie unter Umständen nicht wiedererkennen: Im Sammelband „Arms Control in the 21st Century Between Coercion and Cooperation" konstatieren einige Autoren einen Paradigmenwechsel weg von der verhandelten hin zur erzwungenen Abrüstung. Damit firmierte auch der „Entwaffnungskrieg“ der USA gegen den Irak 2003 unter dem Etikett der Rüstungskontrolle. Grund genug zum Nachforschen: Gibt es tatsächlich einen Formwandel? Was ist von der ursprünglichen Fundamentalkritik, was von den anfänglichen Hoffnungen geblieben? Wo liegen Leistungen und Versäumnisse des Konzepts? Wird es durch neue technologische Entwicklungen überfordert? S+F hat einen Mitherausgeber des Sammelbands, zwei Kontrahenten der ersten Stunde sowie zwei weitere langjährige Kenner der Materie als Autoren gewonnen.
\end{abstract}

Sabine Jaberg

\section{Gibt es einen Formwandel der Rüstungskontrolle?}

\section{Oliver Meier}

Ist die Rüstungskontrolle in der Krise? Auf den ersten Blick scheint der Befund eindeutig: Seit 2010 setzt Russland die Umsetzung des Vertrags über eine Begrenzung konventioneller Waffensysteme - einst ein Kernstück der europäischen Rüstungskontrolle - aus. Der Iran verletzt weiter seine nichtverbreitungspolitischen Verpflichtungen. Der Atomteststopp-Vertrag kann auch 17 Jahre nach Abschluss der Verhandlungen nicht in Kraft treten, weil einige Atomwaffenbesitzer die Unterschrift verweigern. Nordkorea führte jüngst seinen dritten Atomtest durch. Die Genfer Abrüstungskonferenz ist seit 1996 blockiert. Militärische Zukunftstechnologien wie die Raketenabwehr, Informationstechnologie oder bewaffnete Drohnen entziehen sich rüstungskontrollpolitischen Regelungen. Neue Rüstungswettläufe drohen.

Auf den zweiten Blick wirkt das Bild nicht ganz so düster. Die USA und Russland haben im April 2010 den New-Start-Vertrag abgeschlossen und reden weiter über eine Reduzierung ihrer Nukleararsenale. In Europa hat sich die militärische Lage so weit entspannt, dass Obergrenzen auch ohne rechtliche Verpflichtung fast überall eingehalten werden. Nordkorea und Iran versuchten zwar den nuklearen Nichtverbreitungsvertrag (NVV) zu umgehen, wurden aber ertappt. Im Mai 2010 gelang es nach zehn Jahren erstmals wieder, sich auf der Überprüfungskonferenz des NVV auf ein politisch verbindliches Aktionsprogramm zu einigen. Und seit 1997 sind international umfassende Regelungen über das Verbot von Antipersonenminen sowie von Streumunition vereinbart worden. Die UN-Generalversammlung nahm Anfang April mit überwältigender Mehrheit das Abkommen über eine Begrenzung des Waffenhandels an.

Um diese gegenläufigen Entwicklungen zu erfassen, bedarf es eines breiten Begriffs von Rüstungskontrolle. Seit den 1960er
Jahren steht zwar die vertragliche Begrenzung vorhandener militärischer Kapazitäten im Fokus. Dieses enge Rüstungskontrollkonzept taugt aber zur Beschreibung heutiger Praxis nur bedingt. Moderne Rüstungskontrolle umfasst alle Formen der kooperativen Kontrolle militärisch relevanter Technologien, Fähigkeiten und Kapazitäten. War die Rüstungskontrolle früher eine Domäne der beiden militärischen Blöcke, so nehmen heute neue, auch nichtstaatliche, Akteure Einfluss auf ihren Erfolg oder Misserfolg. Unter einem solch weiten Oberbegriff lassen sich gegenwärtig drei Hauptfelder identifizieren:

- Klassische Rüstungskontrolle verfolgt weiterhin einen stabilitätsorientierten Ansatz durch die Regulierung militärischer Potenziale.

- Nichtverbreitungsregime wollen die Gefahr minimieren, dass sensitive Technologien für nicht-friedliche Zwecke missbraucht werden.

- Humanitäre Rüstungskontrolle zielt primär auf eine Verminderung des Gewaltaufkommens während und nach innerstaatlichen Konflikten.

"Coercive arms control“ - etwa im Rahmen von Sanktionen oder anderen Zwangsmaßnahmen - wird hier nicht unter den Begriff der Rüstungskontrolle gefasst. Zwang kann unter den Voraussetzungen der völkerrechtlichen Legitimität, der Angemessenheit und der Effektivität eine wichtige Rolle bei der Durchsetzung konsensual vereinbarter Regeln spielen. Der langfristige Erfolg von Rüstungskontrolle hängt letztendlich aber von der Freiwilligkeit, der Reziprozität und der Inklusivität der eingegangenen Verpflichtungen $a b$.

Klassische Rüstungskontrolle befindet sich in einer Krise, weil ein gleichgewichtsorientierter Ansatz unter den Bedingungen amerikanischer militärischer Dominanz kaum mehr funktioniert. Rund 40 Prozent der globalen Militärausgaben entfielen im Jahr 2011 auf die USA. In den Jahren 2000-2009 betrug das Verhältnis der Militärausgaben der europäischen NATO-Staaten zu denen Russlands 7:1 - unter Einbeziehung 
der USA erhöht es sich sogar auf 19:1. ${ }^{1}$ Das Ungleichgewicht militärischer Fähigkeiten zeigt sich besonders eklatant bei den Zukunftstechnologien wie etwa Raketenabwehrsystemen und modernen konventionellen Waffen großer Reichweite und hoher Präzision. Bei diesen potenziell stabilitätsgefährdenden Systemen haben die USA einen so großen Vorsprung, dass gleichgewichtsorientierte Rüstungskontrolle kaum greifen kann. China und Russland versuchen teils asymmetrisch auf die militärische Dominanz der USA zu antworten. China $(8,2$ Prozent der weltweiten Militärausgaben) investiert zum Beispiel in die Cyberkriegführung, Russland (4,1 Prozent) wertet militärisch weitgehend irrelevante Nuklearwaffen als Symbole strategischer Parität auf. In beiden Fällen drohen neue Rüstungswettläufe oder sind schon in vollem Gange.

Chancen, klassische Rüstungskontrollkonzepte erfolgreich anzuwenden oder wiederzubeleben, dürften daher eher in Regionen bestehen, wo Sicherheitsdilemmata noch heute die Politik bestimmen. Südasien, aber auch der Mittlere Osten gelten oft als Kandidaten. Allerdings erschwert in beiden Regionen die Multipolarität der sicherheitspolitischen Beziehungen eine direkte Übertragung der positiven rüstungskontrollpolitischen Erfahrungen, die unter den Bedingungen der Bipolarität in Europa gemacht wurden.

Für globale Nichtverbreitungsregime stellen die Auswirkungen der Globalisierung die größte Herausforderung dar. Die Diffusion militärisch relevanter Technologien, die Beschleunigung technologischer Entwicklungen sowie die gewachsene Bedeutung neuer Akteure fordern bestehende Nichtverbreitungsregime heraus. Beispiel Chemiewaffenübereinkommen (CWÜ): Anfang der 1990er Jahre noch unter dem Eindruck des OstWest-Konflikts ausgehandelt, spielen Chemiewaffen heute militärisch keine große Rolle mehr. Die Abrüstung bestehender Bestände ist weit vorangeschritten, über drei Viertel der insgesamt mehr als 70.000 Tonnen deklarierter Chemiewaffen - vor allem in den USA und Russland - wurden unter internationaler Kontrolle vernichtet. Mitte 2012 zeigte sich, wie gering der strategische Wert von Chemiewaffen mittlerweile ist. Die Drohung Syriens, auf eine ausländische Intervention in den Bürgerkrieg mit dem Einsatz von Chemiewaffen zu antworten, entpuppte sich als Eigentor. Prompt erklärten westliche Staaten, dass ein solcher Einsatz zwingend eine Intervention zur Folge hätte. Abschreckung geht heute anders.

Künftige Herausforderungen des CWÜ liegen in der effektiven Kontrolle moderner, sich teils rapide entwickelnder Technologien. Zum einen verlagern sich globale Produktionskapazitäten. Mehr als 5.000 hochmoderne chemische Produktionsanlagen sind flexibel auf verschiedene Produkte umstellbar und wären für einen Missbrauch zur Chemiewaffenherstellung technisch besonders gut geeignet. Diese Anlagen befinden sich überwiegend nicht mehr in den Industrieländern des Nordens, sondern vor allem in den Schwellenländern des Südens. Sie werden bisher kaum kontrolliert, auch weil Staaten wie China und Indien Belastungen durch zusätzliche Überprüfungen fürchten. Sie verweisen auf bestehende Inspektionsregeln, die nur im Konsens reformiert werden kön-

1 Alle Zahlen stammen vom Stockholm International Peace Research Institute (SIPRI) nen. Zum anderen ermöglichen moderne chemische (und biochemische) Verfahren die Herstellung neuartiger Agenzien mit eventuell hohem Gefahrenpotenzial. Diese Stoffe werden durch das vorhandene Verifikationsregime aber nur bedingt erfasst. Vergleichbare Probleme bestehen bei der Kontrolle von Technologien, die zur Herstellung biologischer und nuklearer Waffen genutzt werden könnten.

Zugleich eröffnet die Globalisierung auch Chancen. So können zunehmende Vernetzung und neue Informationstechnologien die Verifikation der Vertragstreue verbessern. Und in Ansätzen wird bereits versucht, private Akteure direkt in die effektivere Umsetzung von NV-Regimen einzubeziehen, etwa im Rahmen von Maßnahmen zur Verbesserung biologischer oder nuklearer Sicherheit.

Humanitäre Rüstungskontrolle soll vor allem menschliches Leid minimieren, das durch die Anwendung militärischer Gewalt während und nach innerstaatlichen Konflikten entsteht. Der humanitäre Impetus und das Verbot bestimmter Arten der Kriegführung sind in der Rüstungskontrolle aber keineswegs neu. Bereits nach dem Ersten Weltkrieg wurde der Ersteinsatz chemischer und biologischer Waffen geächtet. Das UN-Waffenübereinkommen verbietet seit den 1980er Jahren besonders grausame Waffen, wie Dum-Dum-Geschosse, Brand- und Blendwaffen.

Das Ottawa-Abkommen von 1997 über das Verbot von Antipersonenminen und das Oslo-Abkommen von 2008 über das Verbot von Streumunition setzten nichtsdestoweniger rüstungskontrollpolitische Meilensteine. Zivilgesellschaftlichen Protagonisten gelang es, neue Diskurse zu etablieren: Nicht mehr die Folgen von Rüstungskontrolle für militärische Handlungsfähigkeit und Stabilität standen im Vordergrund, sondern das durch Waffeneinsatz verursachte menschliche Leid. Der initiierte Normbildungsprozess entpuppte sich als politisch extrem wirksam. Er ermöglichte eine Umgehung des in der Rüstungskontrolle sonst als sakrosankt geltenden Konsensprinzips. Die Verbote wurden gegen den teilweise erbitterten Widerstand einiger militärisch wichtiger Staaten wie China, Russland und die USA durchgesetzt.

Mittlerweile müssen auch Außenseiter, die den Abkommen fernbleiben, ihre Praxis an den in den Verträgen niedergelegten Verhaltensnormen messen lassen. Israels Einsatz von Streumunition im Libanon 2006 wurde international breit verurteilt, obwohl Jerusalem den Oslo-Vertrag nie unterzeichnet hat. Auch die humanitäre Rüstungskontrolle profitiert insofern von der Globalisierung, als sie die weltweite Verbreitung von Normen zu ihrem Vorteil nutzt.

Wie geht es weiter? Rüstungskontrolle bleibt wichtig. Nach wie vor werden jedes Jahr rund 1,7 Billionen US-Dollar für Militär ausgegeben, mehr als 4,6 Milliarden täglich. In vielen Regionen verschärft die Hochrüstung Konflikte zusätzlich. Moderne Waffen sind zielgenauer, drohen damit aber auch die Einsatzschwelle zu senken. Die Zerstörungskraft moderner Technologien ist beispiellos, die Gefahr ihres Missbrauchs, auch durch Terroristen, hoch. Und immer noch wird das meiste Leid durch „ganz normale“ Waffen in vergleichsweise kleinen Konflikten verursacht.

Es gibt also genug Gründe, Rüstungskontrolle weiter zu betreiben - trotz oder gar wegen der vielen neuartigen Heraus- 
forderungen. Selbstverständlich müssen die rüstungskontrollpolitischen Antworten genauso vielfältig ausfallen wie die Problemlagen, die sie regeln sollen. Hier seien nur einige Bausteine moderner Rüstungskontrolle genannt:

- Die militärisch stärksten Staaten müssen zu der Einsicht gebracht werden, dass eine Selbstbeschränkung bei modernen Rüstungstechnologien in ihrem aufgeklärten Eigeninteresse liegt.

- Klassische Rüstungskontrolle bleibt wichtig, aber nicht immer erweist sich ein völkerrechtlich verbindlicher und verifizierbarer Vertrag als das erfolgversprechendste Modell kooperativer Regulierung.

- Bei der Nichtverbreitung gilt es, die Chancen der Globalisierung für eine Kontrolle missbrauchsrelevanter Technologien zu nutzen, indem neue Technologien zur Kontrolle eingesetzt und neue Akteure eimbezogen werden.

- Die normative Wirkung von rüstungskontrollpolitischen Vereinbarungen muss in Bezug auf die Entwicklung, die Produktion, die Weitergabe und nicht zuletzt den Einsatz von Waffen gestärkt werden.

Es gibt also keinen Anlass, sich von der Rüstungskontrolle zu verabschieden, aber viele Gründe, sich Gedanken über die Anpassung vorhandener und die Entwicklung neuer Konzepte und Instrumente zu machen.

Dr. Oliver Meier ist Wissenschaftler in der Forschungsgruppe Sicherheitspolitik an der Stiftung Wissenschaft und Politik (SWP) in Berlin.

\section{Was bleibt von der Fundamentalkritik an der Rüstungskontrolle?}

\section{Dieter Senghaas}

Versteht man unter Fundamentalkritik eine Kritik unter allen Umständen, so hat es sie punktuell gegeben. Sie war aber nicht identisch mit der von mir Ende der 1960er und zu Beginn der 1970er Jahre artikulierten Position. Denn eine Kritik unter allen Umständen erweist sich im Hinblick auf die kooperative Rüstungssteuerung - auch Rüstungskontrolle (arms control) genannt - als nicht sinnvoll. Zweifelsfrei besitzt eine genuine kooperative Rüstungssteuerung einen positiven funktionalen Stellenwert, wenn sie tatsächlich mäßigend bzw. abbauend in einen Rüstungswettlauf eingreift. Dabei spielt es keine Rolle, ob dieser Rüstungswettlauf das Ergebnis von tatsächlichen AktionsReaktionsprozessen zwischen den Kontrahenten ist oder eher einer überwiegend innenpolitisch verursachten einpoligen, zweibzw. mehrpoligen Rüstungsdynamik entspringt. Entscheidend ist aus programmatischer Perspektive, ob Rüstungsdynamik abgefedert bzw. abgebaut wird, sodass alte Waffensysteme tendenziell beseitigt und neue nicht disloziert werden. Abrüstung stellt dann eine erweiterte Zielsetzung dar. Erforderlich bleibt überdies natürlich der Aufbau einer Friedensstruktur, in der bleibende Interessen- und Identitätskonflikte selbstverständlich gewaltfrei bearbeitet werden. Die Androhung oder gar Anwendung militärischer Gewalt wären dann strukturell überwunden.
Ob kooperativer Rüstungssteuerung in einem erwartbar langwierigen Übergangsprozess von konfrontativer Rüstungsdynamik zu einer nachhaltigen Friedensstruktur ein positiver Stellenwert zukommt, ist somit nicht eine prinzipielle, sondern eine empirische Frage. Sie kann ausschließlich mit Blick auf die je vorliegenden konkreten Sachverhalte beantwortet werden.

In meinem Buch „Aufrüstung durch Rüstungskontrolle“ (Stuttgart 1972) reagierte ich konkret auf drei Abkommen, die im Mai 1972 zwischen den USA und der Sowjetunion vereinbart wurden: 1) der Vertrag über die Begrenzung der Anti-Ballistic-Missile-Systeme (ABM-Vertrag), 2) das Interimsabkommen über die Begrenzung von Offensivwaffen sowie 3) das Protokoll zum Interimsabkommen über die Begrenzung von Offensivwaffen. Das Bemerkenswerte bei diesen sogenannten SALT-Abkommen bestand damals darin, dass zwar überkommene offensive und defensive Rüstungsvorhaben auf nuklearstrategischer Ebene quantitativ begrenzt wurden (so die Zahl der dislozierbaren ABM-Systeme sowie die Zahl der landgebundenen und der seegestützten Offensivsysteme). Gleichzeitig jedoch wurden die sich seit Mitte der 1960er Jahre abzeichnenden neuen qualitativen Rüstungstechnologien, insbesondere die MIRV-Technologie der Mehrfachsprengköpfe bewusst und willentlich von diesen Abkommen nicht erfasst. Die Pointe bestand darin, dass der ABM-Vertrag in Artikel VII festlegte: „Vorbehaltlich der Bestimmungen dieses Vertrages darf eine Modernisierung und ein Ersatz von ABM-Systemen oder ihrer Komponenten vorgenommen werden. " Und im Interimsabkommen über bestimmte Maßnahmen hinsichtlich der Begrenzung von strategischen Offensivwaffen hieß es in Artikel IV entsprechend: „Vorbehaltlich der Bestimmungen dieses Interimsabkommens darf eine Modernisierung und ein Ersatz strategischer ballistischer Offensivraketen und von Abschußvorrichtungen, die von diesem Interimsabkommen erfaßt werden, vorgenommen werden".

Wer einigermaßen mit den rüstungstechnologischen Entwicklungen vertraut war, konnte bereits damals wissen, was dann später eingetreten ist: Diese Abkommen, vor allem das Interimsabkommen, legten zwar quantitative Begrenzungen fest. Sie stellten aber im Grunde genommen einen völkerrechtlichen Freibrief für eine qualitative Um- bzw. Aufrüstung dar. Um nur einen maßgeblichen Sachverhalt herauszugreifen: Man begrenzte die landgebundenen und die seegestützten Interkontinentalraketen, ersetzte sie im Rahmen der quantitativen Begrenzung zum Teil durch neue und bestückte sie mit Mehrfachsprengköpfen so viel man wollte - zunächst mit drei, später mit bis zu sechzehn Sprengköpfen pro Einheit.

Rüstungskontrolle fand also statt, aber sie eröffnete einen von den Vertragspartnern vereinbarten Weg zu einer qualitativen Um- und Aufrüstung. Sie erwies sich daher im Hinblick auf die eingangs zitierte Programmatik einer genuinen kooperativen Rüstungssteuerung als widersinnig. Sie entpuppte sich sogar als konterproduktiv, weil die Ermöglichung neuer qualitativer Rüstungsschübe innerhalb der beiden Vertragspartner, USA und Sowjetunion, den Rüstungswettlauf zwischen ihnen zwangsläufig intensivieren musste.

Die hier umrisshaft dargestellten Vorgänge vor nunmehr etwas mehr als vierzig Jahren lieferten ein eindrucksvolles Lehrstück 
für eine erfahrungswissenschaftliche Auseinandersetzung mit Rüstungskontrollverhandlungen und Rüstungskontrollabkommen innerhalb der Friedensforschung wie auch der Disziplin der Internationalen Beziehungen. Diese Abkommen und Verhandlungen gilt es, damals nicht anders als heute, immer im Kontext der tatsächlichen und emergenten Rüstungsdynamik zu analysieren. Es ist seit Jahrzehnten kein Geheimnis mehr, dass Rüstungsdynamik sich aus einem Zusammenwirken unterschiedlicher Faktoren ergibt. Hier gehen geopolitische Überlegungen und nationalstaatliche Interessen ebenso wie Bedürfnisse der Teilstreitkräfte und industrielle Interessen ein. Hinzu kommen rüstungstechnologische Entwicklungen, wie sie von allen Beteiligten, insbesondere auch von Wissenschaftlern, vorangetrieben werden. Des Weiteren spielen programmatische und ideologisch-politische Orientierungen eine Rolle. Mithin handelt es sich um eine redundante Verursachung, derzufolge der eine oder andere Faktor zeitweilig abgeschwächt wirken kann, ohne das Gesamtgebilde der Rüstungsdynamik wirklich zu beeinträchtigen. Auch fällt auf, dass Rüstungsdynamik ab einer bestimmten Größenordnung bei sämtlichen Konfliktbeteiligten einem Wettlauf mit sich selbst gleicht. Sie wird also selbstreferenziell bzw. autistisch. Dieser Sachverhalt ist aus der Konfliktanalyse im Allgemeinen bekannt. Er tritt besonders dann auf, wenn vielfältige Faktoren, Impulse und Interessen in eine Konfliktdynamik einfließen und diese bestimmen. Unter solchen Umständen erweisen sich steuernde Eingriffe als besonders schwierig. Rüstungskontrolle nimmt dann jene Funktion an, die Murray Edelman in anderem Zusammenhang "the symbolic uses of politics" genannt hat.

Mein damaliger Buchtitel „Aufrüstung durch Rüstungskontrolle" wurde vielfach als polemisch wahrgenommen. Er erwies sich aber seinerzeit und im Lichte der nachfolgenden Entwicklungen auf der nuklearstrategischen Ebene als empirisch wohlbegründet. Sind die Sachverhalte hinsichtlich der jüngsten Rüstungskontrollabkommen auf nuklearstrategischer Ebene (SORT/ NEW START) gänzlich andere oder ähneln sie den Erfahrungen von 1972? Welche einschlägigen Sachverhalte liegen Inszenierungen kooperativer Rüstungssteuerung in anderen Bereichen zugrunde? Es wäre wünschenswert, wenn Friedensforscher und Friedensforscherinnen der jüngeren Generation sich einmal um ein umfassendes erfahrungswissenschaftlich fundiertes Urteil bemühen würden, um absehbare Entwicklungstrends in allen bestehenden und sich neuerdings herausbildenden Rüstungsbereichen (cyberwar) im Lichte der Erfahrungen mit kooperativer Rüstungssteuerung in den vergangenen Jahrzehnten fair beurteilen zu können.

Prof. Dr. Dieter Senghaas ist Senior Fellow am Institut für Interkulturelle und Internationale Studien (InIIS), Universität Bremen.

\section{Was bleibt von den Hoffnungen auf die Rüstungskontrolle?}

\section{Erhard Forndran}

Eine Bemerkung vorweg: Es scheint kaum möglich, die aufgeworfene Frage im knappen Forumsformat umfassend zu be- antworten. Allenfalls ist es machbar, einige für das Verständnis der Wirkungsgeschichte der Rüstungskontrolle wesentlichen Gesichtspunkte in Form von inhaltlich nicht näher begründeten Thesen vorzustellen. Der Autor will nicht verschweigen, dass er bei diesem Vorgehen ein schlechtes Gewissen gegenüber dem Leser hat.

Eine zweite Bemerkung vorweg betrifft den Gebrauch der Begriffe. Die folgenden Ausführungen sprechen nicht von „kooperativer Rüstungssteuerung“, sondern von „Rüstungskontrolle“. Nicht nur kooperative Formen, sondern auch einseitige oder gradualistische Schritte zur Förderung der Stabilität internationaler Beziehungen sind denkbar. Festzuhalten ist außerdem, dass der Begriff „Kontrolle“ nicht primär die Einhaltung eines Zustandes meint - das zwar auch, aber vor allem geht es um die Steuerung eines dynamischen Prozesses nach Stabilitätsgesichtspunkten.

Welche Wurzeln besitzt die Rüstungskontrolle? Mehrere Gesichtspunkte sind zu berücksichtigen. Ein erster Ausgangspunkt verwarf die These, durch Abrüstung könne Frieden geschaffen werden. Die Bewertung hochkomplexer Faktoren - der demografischen, geografischen, wirtschaftlichen, wissenschaftlichen und politischen Realitäten - führe nicht zu stabilitätskonformen, die rüstungsrelevanten technischen Entwicklungen berücksichtigenden Konzepten für den Abrüstungsprozess und für die Gewährleistung des abgerüsteten Zustandes. Die Erfahrungen zeigten, dass Wiederaufrüstung und Rüstungswettlauf jederzeit möglich und neue Ungleichgewichte zugunsten der ökonomisch-technologisch überlegenen Staaten zu erwarten seien.

Die zweite Wurzel des Rüstungskontrollkonzeptes ergab sich aus der Tatsache, dass die erste Generation von Kernwaffen zwar großen Schaden anrichten konnte, aber keine ausreichende Überlebenschance bei einem gegnerischen Angriff besaß. Es fehlte die Abschreckungsfähigkeit. Überlebens- und Zweitschlagfähigkeit bildeten aus Sicht der Rüstungskontrolle jedoch die erforderliche Bedingung einer glaubwürdigen Friedenssicherung durch Abschreckung. Rüstungskontrolle ist weder mit Abrüstung noch mit Abschreckung identisch. Sie kann aber für beide Konzepte Vorschläge zur Stabilitätsförderung unterbreiten. Diese können zur Aufrüstung, Umrüstung oder Abrüstung führen und damit unterschiedliche Denkschulen hervorbringen: Stabilisierung der Abschreckung oder Reduzierung auf Minimalabschreckung. In Bezug auf Abrüstung kann sie Vorstufe und Durchführungsbedingung sein, in Bezug auf Abschreckung vermag sie dazu beitragen, dass ein Staat mit Zweitschlagfähigkeit in der Krise abwarten kann.

Eine dritte Wurzel ging von der Einsicht aus, dass Kommunikationswege innerhalb eines Staates und zwischen den Staaten die Gefahren eines Überraschungsangriffes oder einer Eskalation beeinflussen können. Als besondere Risiken galten sowohl ein Mangel als auch ein Übermaß an Informationen, aber auch Fehlinterpretationen und wirklichkeitsferne Risikokalküle. Dementsprechend wurde die Einrichtung bzw. Verbesserung von Kommunikationswegen auch zwischen Kontrahenten und in Krisen gefordert, um ungewollten Eskalationen besser vorbeugen zu können. Gleichzeitig sollte sichergestellt 
sein, dass nur Befugte den Einsatz bestimmter - insbesondere atomarer - Waffen anordnen konnten.

Viertens gewann allmählich die Frage an Relevanz, auf welchem Wege sich andere als militärisch dominierte Verhaltensweisen in den Vordergrund der Politik rücken ließen. Rüstungskontrolle wurde zum Beurteilungskriterium für die Entspannungspolitik und zugleich zum Teil dieser Politik. Am KSZE-Prozess ließ sich dies nachvollziehen. Die Rüstungskontrolle wurde politischer und förderte die Erwartungsstabilität. Unilaterale und gradualistische Rüstungskontrolle, Krisenmanagement und eine Politik regionaler Stabilitätsmaßnahmen wurden möglich. Bedeutung kam dabei der Einsicht zu, dass die Verbesserung der Sicherheitslage des Gegners auch der eigenen Sicherheit dienen konnte. Dies zeigte sich u.a. im SALT-I-Vertrag mit seinem weitgehenden Verzicht auf Raketenabwehrraketen.

Wo liegen nun Erfolge und Grenzen der Leistungsfähigkeit der Rüstungskontrolle? Sie hat zweifellos in der Zeit von der KubaKrise bis zum Ende des Ost-West-Konfliktes eine erhebliche Rolle gespielt. Auf die Liste nennenswerter Ergebnisse gehören neben der Verbesserung der Kommunikationswege auch der Teststopp-Vertrag, der Vertrag über die Nichtweiterverbreitung von Kernwaffen und der Prozess, der zur Null-Lösung bei den Mittelstreckenraketen und zu den SALT/START-Regelungen führte. Als bedeutsam erwiesen sich außerdem das wachsende Verständnis für das Denken des Anderen und die zunehmende Erwartungsstabilität.

Trotzdem stand das Konzept der Rüstungskontrolle von Anfang an in der Kritik. Manche Einwände durften eine gründliche Prüfung der ihnen zugrundeliegenden Argumente erwarten. Als besonders fruchtbar erwiesen sich jene Fälle, in denen es zwischen den konkurrierenden Interpretationen $\mathrm{zu}$ begründeter Kritik und Kritik der Kritik kam. Die Befürworter der Rüstungskontrolle mussten eingestehen, dass diese sich primär auf die militärische oder technologisch bedingte Instabilität konzentrierte. Zweifellos ging von der Verhinderung des nuklear geführten Krieges auch die Gefahr aus, dass die Wahrscheinlichkeit kleiner Kriege - Stellvertreterkriege, konventionelle Kriege oder Bürgerkriege - wegen des verringerten Risikos zunahm. Es wurde auch kritisch angemerkt, dass die häufige Gleichsetzung der Menge vorhandener Rüstung mit Stabilität nicht sachgerecht sei, weil die qualitative Seite von Rüstungen übergangen werde. Die Rüstungskontrolle hat im Übrigen schon immer vor der Gefahr gestanden, dass die Friedenssicherung durch Abschreckung in eine Erzwingungsstrategie übergehen konnte. Abschreckung will ein Nichthandeln erreichen, Erzwingung im Gegenteil (eigenes) Handeln ermöglichen.

Das Funktionieren der Abschreckung unter den Bedingungen der Rüstungskontrolle hängt auch von der Glaubwürdigkeit der Schadensandrohung gegenüber einem potenziellen Angreifer und der Wahrscheinlichkeit einer tatsächlichen Anwendung der militärischen Option ab. Je kleiner der angedrohte Schaden durch die Aktionen des Angegriffenen, umso größer die Wahrscheinlichkeit einer Aggression. Je größer der angedrohte Schaden, umso geringer die Glaubwürdigkeit der Reaktion. Umfang des möglichen Schadens und die Wahr- scheinlichkeit des Handelns müssen hier also sicherheitspolitisch zusammen gedacht werden.

Welche Auswirkungen haben die „neuen Herausforderungen“ auf die Rüstungskontrolle? Erstens muss der Annahme, die altbekannten Herausforderungen seien nicht mehr relevant, widersprochen werden. Die Nichtweiterverbreitung von Kernwaffen, die Raketenabwehrraketen-Systeme, die militärische Nutzung des Weltraumes und der konventionelle Rüstungswettlauf sowie der ganze Bereich des Krisenmanagements und der Vertrauensbildenden Maßnahmen bleiben wichtige Felder einer Politik, die Gewaltanwendung möglichst verhindern will. Es geht also nicht um die Neuerfindung der Rüstungskontrolle, sondern um eine Ergänzung bzw. Erweiterung bestehender Ansätze.

Ein erster Schritt bei der Erarbeitung eines erweiterten Rüstungskontrollkonzeptes muss die Veränderungen der Akteursebene bedenken. Die klassische Rüstungskontrolle bezog sich auf die Beziehungen zwischen Staaten, primär auf diejenige zwischen den beiden Weltmächten. Mittlerweile geht es aber um mehr Staaten und um nichtstaatliche Gewaltakteure. Vor allem innerstaatliche und innergesellschaftliche Realitäten sind für wachsende, auch grenzüberschreitende Instabilitäten verantwortlich. Fragile Staatlichkeit wird anfällig für Kriminalität, Terrorismus und Bürgerkriege. Technische Fähigkeiten eröffnen die Möglichkeit des cyberwar. Ideologische und kulturelle Faktoren verschärfen die Konfrontation mit anderen Weltbildern und Wertesystemen. Nur einige Hinweise sollen die wachsende Themenfülle verdeutlichen: Der Waffenhandel, besonders mit Kleinwaffen, der Einsatz von Kindersoldaten und die Herrschaft von warlords betreffen nicht nur die Durchsetzung der Menschenrechte, sondern sind auch notwendige Handlungsfelder künftiger Stabilitätspolitik. In welchem Umfang eines Tages auch nichtstaatliche Akteure über Massenvernichtungswaffen verfügen werden und ob daraus besondere Gefahren erwachsen, ist umstritten. Umstritten ist auch, in welchem Umfang unilaterale und gradualistische Rüstungskontrolle zur Beherrschung krisenartiger Konstellationen beitragen können, indem sie etwa Koalitionsbildung, Kompromisssuche und Konsensfindung unterstützen und Konflikte verregeln helfen. Ebenfalls umstritten ist, welche Haltung die Rüstungskontrolle gegenüber den sogenannten Abrüstungs- und Erzwingungskriegen einnehmen soll.

Zusammenfassend kann nicht von einem Versagen der Rüstungskontrollphilosophie in der Vergangenheit gesprochen werden, sehr wohl aber von einer unterschiedlichen Bedeutung ausgehandelter Abkommen. Auch von einem Bedeutungsverlust klassischer Themen kann nicht die Rede sein, von Unklarheiten in Bezug auf die Erfolgswahrscheinlichkeit angesichts neuer Herausforderungen hingegen schon. Eine politisch motivierte Beendigung der Rüstungskontrollpolitik durch die heute wichtigen Akteure ist ebenfalls nicht zu konstatieren, wohl aber berechtigte Ungewissheit in Bezug auf die Einbindung neuer Gewaltakteure.

Die Forderungen der Rüstungskontrolle an die Politik sind gut begründet, ihre Erfolge in der Praxis aber nur für den Einzelfall zu beurteilen. Dementsprechend erlauben die Erfahrungen weder ein eindeutiges noch ein allgemeingültiges Urteil über die 
Bedeutung dieses Konzeptes. Gewicht besaß es, als es um die Beherrschung der technischen Merkmale der Rüstungen und die Gefahr politisch nicht gewollter Kriege beziehungsweise ungewollter Eskalationen ging. Erschwert wird die Urteilsbildung durch die Tatsache, dass Rüstungskontrolle kein beendeter Prozess ist, sondern Daueraufgabe bleiben wird. Der Wandel der Gründe und der Formen sowie die Vielfalt möglicher Ergebnisse erlauben nur ein begrenztes Lernen aus früheren Erfahrungen. Aus der Kontextabhängigkeit aller stabilitätsorientierter Maßnahmen ergeben sich weitreichende Fragen: Wie sehen die Grundmerkmale einer stabilen politischen Ordnung aus? Welche Rolle können demokratische Systeme spielen? Und wie ist mit dem Spannungsverhältnis von Gewaltverbot und Interventionsrecht zum Schutz der Menschenrechte umzugehen? Eine Beantwortung dieser und anderer Fragen kann sich nicht auf die Position zurückziehen, den Umfang des Themenkatalogs der Rüstungskontrollpolitik enger zu fassen. Sie muss sich der Komplexität des Themas stellen.

Prof. Dr. Erhard Forndran ist Emeritus des Instituts für Politikwissenschaft der Otto-von-Guericke-Universität Magdeburg.

\section{Wo liegen Leistungen und Versäumnisse der Rüstungskontrolle?}

\section{Götz Neuneck}

Moderne Rüstungskontrolle entstand während des Kalten Krieges vor dem Hintergrund der steigenden Gefahr der globalen Auslöschung des Planeten durch Nuklearwaffen. Man kann sie ganz einfach als internationale Kontrolle aller Art von Rüstungen für Kriegszwecke durch die Begrenzung oder das Verbot von bestimmten Waffen und deren Einsatz begreifen. Sie bezieht sich auf den gesamten Waffenzyklus, der von der Herstellung über den Besitz bis hin zur Weitergabe oder den Einsatz reicht. Die Verpflichtung zur Zerstörung geächteter Waffen kann hinzukommen. Rüstungskontrollübereinkommen vermögen unterschiedliche Ziele im Rahmen des Konfliktspektrums abzudecken. Sie können präventive Funktion übernehmen, indem sie zur Gefährdungsminimierung oder Kriegsverhütung beitragen, Krisenmanagement („Rotes Telefon“) stärken oder durch kooperative Vereinbarungen das wechselseitige Rüstungsverhalten von Antagonisten beeinflussen. Der Begriff „kooperative Rüstungssteuerung“ verdeutlicht dies in besonderer Weise. Rüstungskontrolle lässt sich aber auch zur und nach Beendigung eines Konflikts nutzen, um die Bedingungen nach einem Waffenstillstand festzulegen (z.B. die UNO-Resolution 687 nach Ende des Zweiten Golfkriegs 1991 oder auch das Dayton-Abkommen im Kontext der Jugoslawienkriege 1995).

Die Leistungen der Rüstungskontrolle können sich sehen lassen: Herstellung, Besitz, Weitergabe und Einsatz von chemischen und biologischen Waffen sind heute völkerrechtlich geächtet. Im Falle der Nuklearwaffen besteht zwar ein solches Verbot nicht; allerdings hat der Internationale Gerichtshof am 8. Juni 1996 in Den Haag erklärt, dass die Androhung und der Einsatz von Atomwaffen grundsätzlich gegen diejenigen Regeln des Völkerrechts verstoßen würden, die für bewaffnete
Konflikte gelten, insbesondere das humanitäre Kriegsvölkerrecht. Er unterstreicht das Tabu, das seit den Abwürfen auf Hiroshima und Nagasaki existiert.

Die Zahl nuklearer Sprengköpfe hatte 1987 die irrsinnige Höhe von 80.000 erreicht, seither nimmt sie jedoch jährlich ab. Heute gelten noch ca. 20.000 Sprengköpfe im Besitz der fünf Nuklearwaffenstaaten (USA, Russland, China, Großbritannien und Frankreich) und der vier De-facto-Nuklearmächte (Indien, Pakistan, Israel und Nordkorea) als operativ einsetzbar. Die Obergrenze des letzten bilateralen New-Start-Vertrags zwischen den USA und Russland beträgt 1.550 Sprengköpfe pro Seite. Mit Blick auf das Ziel einer Welt ohne Nuklearwaffen gilt diese Zahl gemeinhin als zu hoch. Zwar diskutieren die beiden nuklearen Führungsmächte weitere Reduktionen ihrer strategischen wie taktischen Arsenale, allerdings gilt es, die „kleineren Nuklearwaffenstaaten“ mittelfristig einzubeziehen. Im Laufe der Jahrzehnte wurden nach zähen Verhandlungen Verträge geschlossen, die das horizontale und vertikale nukleare Wettrüsten einschränken sollten. Heute existieren insbesondere in der südlichen Hemisphäre stabile nuklearwaffenfreie Zonen, so in der Antarktis (1959), dem Weltraum (1967), in Lateinamerika (1967), auf dem Meeresboden (1971), im Südpazifik (1985), in Südostasien (1995), in Afrika (1996) und Zentralasien (2006).

Abrüstung und Rüstungskontrolle werden häufig synonym benutzt. Abrüstung bezeichnet die Reduktion oder vollständige Eliminierung bestimmter Waffenkategorien. Ihre Zerstörung erfolgt oftmals im Rahmen von Rüstungskontrollverträgen - etwa dem Chemiewaffenübereinkommen von 1993. Drastische konventionelle Abrüstung fand insbesondere nach Ende des Zweiten Weltkriegs und des Kalten Kriegs statt. Im Rahmen des 1992 in Kraft getretenen KSE-Vertrags wurden ca. 70.000 Stück vertraglich begrenztes Gerät zerstört. Zu diesem Zweck wurde ein umfassendes Begrenzungs-, Informations- und Verifikationssystem geschaffen. Auch der Katalog einschränkender Maßnahmen, die sich im Rahmen von Rüstungskontrolle herausgebildet haben, stellt eine wertvolle Errungenschaft dar. Er umfasst verschiedenartige Maßnahmen: deklaratorische (Anmeldung von Übungen), geografische (entmilitarisierte Zonen), strukturelle (Defensivorientierung) und operative (Manöverbegrenzungen).

Des Weiteren haben sich wirkungsvolle Instrumente und Verfahren zur Vertragseinhaltung herausgebildet. Satellitenverifikation durch nationale technische Mittel, Verdachtsinspektionen oder Sicherungsmaßnahmen (safeguards) sind inzwischen vielfach erprobt. Im Rahmen von Rüstungskontrollverträgen entstanden Überprüfungsorganisationen wie die „Organisation für das Verbot chemischer Waffen" (OVCW) in Den Haag und die „Internationale Atomenergieorganisation“ (IAEO) in Wien. In festgelegten Abständen finden zum Nichtverbreitungsvertrag (NVV) von 1968 sowie dem B- und dem C-Waffenübereinkommen Überprüfungskonferenzen statt, die die Vertragseinhaltung prüfen und Vorschläge zur Verbesserung des Regimes erörtern oder beschließen. In ihrem Umfeld beteiligen sich Nichtregierungsorganisationen, Forschungsinstitutionen und Aktivistengruppen an der Diskussion. Bi- oder multilaterale Rüstungskontrollforen etwa auf UNO- oder OSZEEbene ermöglichen einen ständigen Meinungsaustausch über krisenhafte Entwicklungen und Abrüstungsinitiativen. 
Insbesondere der Abschluss des INF-Vertrages 1987 kündigte das Ende des Kalten Kriegs an. Er führt zur kompletten Abrüstung von zwei Kategorien nuklear bestückter Mittelstreckensysteme („Nulllösung“). Der bereits erwähnte KSE-Vertrag und die beiden START-Verträge $(1991,1993)$ bildeten zwar anfangs kontrovers diskutierte, dann aber weitgehend akzeptierte Rahmen, um eine friedliche Transformation der hochgerüsteten Militärblöcke einzuleiten und später abzusichern. Sie orientieren sich an der Erreichung der gleichen Zahl von Waffensystemen pro Militärbündnis („Paritätsprinzip“). Im Minimalfall kann dies gegenseitiges Verständnis, Deeskalation und Krisenprävention begünstigen sowie Transparenz und Vertrauen zwischen verfeindeten Staaten fördern.

Den Leistungen stehen aber auch entscheidende Versäumnisse gegenüber. Kritiker bemängeln zu Recht eine zu geringe Reichweite in dem Sinne, dass Rüstungskontrolle integraler Bestandteil herkömmlicher Außenpolitiken und Militärdoktrinen bleibt. Die nukleare Rüstungskontrolle zementiert eklatante Asymmetrien: So erlaubt der NVV den bereits etablierten Atommächten den Besitz von Nuklearwaffen, während er ihn allen anderen Staaten untersagt. Damit trägt er zu Proliferationsdynamiken z.B. im Nahen und Mittleren Osten oder in Asien bei. Der Einsatz von Nuklearwaffen ist genauso wenig vertraglich eingeschränkt wie die Weiterentwicklung neuer Nuklearwaffen und Trägersysteme. Zwischen Indien und Pakistan findet ein ungehemmter Rüstungswettlauf bei nuklear bestückbaren Trägersystemen statt. Auch das künftige Verhalten Chinas im pazifischen Raum bleibt unklar. Die technologische Eigendynamik wird bislang nicht eingefangen: Dies zeigt sich besonders deutlich bei der Raketenabwehr sowie bei zielgenauen konventionellen und unbemannten Trägersystemen. Vorbeugende Verbote bei neuen Kategorien wie Laser-, Weltraum- oder Cyberwaffen fehlen. Die Automatisierung des Kriegs, die die militärtechnische Überlegenheit der Führungsmacht USA erhalten soll, schreitet ungehemmt voran. Qualität tritt an die Stelle von Quantität und heizt dadurch gerade asymmetrische regionale Konfliktkonstellationen an. Der raschen Verbreitung von Kleinwaffen wird nicht wirksam Einhalt geboten. Der Zusammenbruch schwacher Staaten, die über erhebliche Militärarsenale verfügen, droht gefährliche Waffen in die Hände substaatlicher Akteure zu spülen.

Rüstungskontrolle erzeugt zudem selber Widersprüche. Bewusst schwach formulierte Verbotstatbestände erlauben militärtechnische Umgehungen oder eröffnen gar Aufrüstungsspielräume. Sie kann zwar Bedingungen für weitere Abrüstung verbessern, nicht jedoch den Rahmen für eine neue Friedensordnung schaffen. Auch fußt klassische Rüstungskontrolle auf dem Paritätsprinzip, ohne klare Kriterien für minimale Obergrenzen vorzugeben. Überprüfungsmöglichkeiten internationaler Verifikationsagenturen sind schwach institutionalisiert und bleiben auf die Unterstützung der Mitgliedstaaten angewiesen. Beispielsweise gelang es auf UNO-Ebene bislang nicht, eine internationale Weltraumagentur zu gründen, die unabhängig von den nationalen Mitteln potenter Staaten Luft- und Satellitenbildverifikation betreiben könnte.

Rüstungskontrolle, insbesondere wenn sie zu Zahlenspielen verkommt, droht Möglichkeiten unmittelbarer Konfliktbeilegung zu überdecken. Letztlich sind nicht die Waffen die
Ursache von Konflikten, die sich vielmehr aus Angst und Misstrauen speisen. Der Mittlere Osten erlebt zurzeit eine neue Rüstungswelle, statt robuste Dialogforen und kollektive Mechanismen zur Streitbeilegung und Krisenbewältigung zu schaffen. Wesentliche Ansätze kooperativer Sicherheit in Europa werden wie der KSE-Vertrag verwässert und geben auch deshalb kein zugkräftiges Modell für andere Krisenregionen ab. Bisher fehlt insbesondere bei den Führungsmächten der politische Wille, Rüstungskontrolle und Abrüstung an die sich entwickelnde Weltordnung des 21. Jahrhunderts anzupassen und in Krisenregionen zu etablieren. Selbstbeschränkung, Vertrauensbildung, Krisenstabilität und die Schaffung neuer Sicherheits- und Friedensstrukturen sind entscheidende Faktoren. Sie erfordern mehr politische Aufmerksamkeit aller betroffenen Staaten, um neue Gewaltexzesse zu verhindern. Solange Rüstungskontrolle nicht sämtliche dieser Ebenen einbezieht, bleibt auch das Risiko der Auslöschung der modernen Zivilisation durch Massenvernichtungswaffen bestehen.

Prof. Dr. Götz Neuneck ist stellvertretender Direktor des Instituts für Friedensforschung und Sicherheitspolitik an der Universität Hamburg (ISFH) und Leiter der Interdisziplinären Forschungsgruppe Abrüstung, Rüstungskontrolle und Risikotechnologien (IFAR ${ }^{2}$ ).

\section{Überfordern neue Technologien die Rüstungskontrolle?}

\section{Jürgen Altmann}

Fast immer ist Rüstungskontrolle neuen Militärtechniken hinterhergelaufen. Wo es aber gelang, sie zu vereinbaren, hat sie im Wesentlichen funktioniert. Eine entscheidende Bedingung für das Zustandekommen vertraglicher Begrenzungen der Militärpotenziale war und ist die Möglichkeit, bei den Vertragspartnern und potenziellen Gegnern die Einhaltung zu überprüfen. Und das so zuverlässig und rechtzeitig, dass Verletzungen der Bestimmungen nicht zu bedrohlichen Asymmetrien bzw. Instabilitäten führen. Die Aussicht, eine Umgehung nicht lange geheim halten zu können, wirkt auf mögliche Übeltäter schon im Vorfeld abschreckend.

Das Erfordernis einer wirksamen Verifikation eingegangener Verpflichtungen erwies sich anfangs als Stolperstein für das Zustandekommen von Rüstungskontrollverträgen, da die Sowjetunion Inspektionen gegenüber sehr misstrauisch war. Einen ersten Durchbruch brachten in den 1960er Jahren die Überwachungssatelliten, die als „nationale technische Mittel der Verifikation" ausreichten, um die Einhaltung von Begrenzungen bei strategischen Kernwaffenträgern (Bombern, Land- und UBoot-Raketen) zu überprüfen. Der zweite Durchbruch kam mit Michail Gorbatschow in den 1980er Jahren. Nun wurden VorOrt-Inspektionen standardmäßig vereinbart - zunächst beim INF-Vertrag über landgestützte atomare Mittelstreckenraketen, dann beim KSE-Vertrag über konventionelle Streitkräfte in Europa. Es folgten START I zu strategischen Trägersystemen für Nuklearwaffen, das Chemiewaffenübereinkommen und der umfassende Teststoppvertrag. Beim Biologiewaffenübereinkommen (BWÜ) von 1972 jedoch gab es Probleme. Bemühungen, das umfassende Verbot solcher Waffen durch ein Ve- 
rifikationsprotokoll zu ergänzen, scheiterten 2001 daran, dass vor allem den USA Inspektionen in militärischen und zivilen Forschungslabors nicht akzeptabel erschienen.

Nach Ende des Kalten Kriegs wurden militärische Forschung und Entwicklung (FuE) nur wenig reduziert. Beide sind jetzt wieder in vollem Schwung. Dabei liegen die USA klar in Führung: Sie tragen zwei Drittel der weltweiten Aufwendungen. Stichworte der letzten Jahre sind netzwerkzentrierte Kriegführung, Präzisionsangriffe und unbemannte Kampffahrzeuge. Forschung findet in voller Breite statt, u.a. für Quanteneffekte, Schnittstellen zu Nerven und Gehirn und magnetohydrodynamische panzerbrechende Munition. Nanotechnik und mit ihr konvergierende Techniken wie Informatik oder Biotechnik eröffnen grundsätzlich neue Möglichkeiten, an deren militärischer Nutzung bereits gearbeitet wird. Absehbar sind metallfreie Schusswaffen sowie Kleinstroboter und -flugkörper. Gleiches gilt für selektive biochemische Waffen, die die Gefahr eigener Ansteckung stark verringern und somit militärisch attraktiver würden. Vieles, was die USA als erstes einführen werden, wird kurz danach auch anderen Ländern möglich sein.

Die nächste Runde des Wettrüstens zeichnet sich bei den bewaffneten Drohnen ab. Inzwischen besitzen etwa 80 Länder unbewaffnete Exemplare. Die Anzahl der Staaten, die über bewaffnete Versionen verfügen, könnte schnell von jetzt vier oder fünf auf zwanzig bis fünfzig steigen. Unbemannte Landund Wasserkampffahrzeuge würden folgen. Westliche Länder hätten kein Monopol mehr, sondern würden von möglichen Gegnern mit großen und kleinen unbemannten Kampfflugzeugen bedroht, Terroristen könnten ausgefeilte kleinere Varianten für Anschläge nutzen. Die Durchführung autonomer Angriffe ohne Entscheidung eines Menschen wird schon angedacht. Hier wird es erheblichen militärischen Druck geben, damit das eigene System auch bei Ausfall oder Störung der Fernsteuerverbindung genutzt oder schnellstmöglich auf einen Angriff reagiert werden kann.

Bei militärischer FuE verfolgen die USA ausdrücklich das Ziel, „bezahlbare und entscheidende militärische Überlegenheit zu ermöglichen, um jeden Gegner auf jedem Schlachtfeld zu besiegen." 2 Das ist aus rein militärischer Sicht stimmig, aber angesichts potenter Rüstungskonkurrenten nicht erreichbar. Man denke nur an einen Atomkrieg oder an einen Krieg gegen eine Koalition großer Staaten und daran, dass Chinas militärische Möglichkeiten mit seiner wirtschaftlichen Stärke deutlich wachsen werden. Mithin ist die Ausrichtung der USA ein Rezept für Destabilisierung und Wettrüsten. Zum Glück haben sie aber auch eine gewisse Tradition, gemeinsam mit anderen Staaten überprüfbare Beschränkungen für die jeweils eigenen Streitkräfte zu vereinbaren. Das gilt sogar für präventive Begrenzungen bei Entwicklung und Erprobung - vor allem bei biologischen und chemischen Waffen, bei Nukleartests und bis 2002 auch bei der Raketenabwehr.

Wegen ihrer führenden Position bei neuer Militärtechnik spielen die USA eine entscheidende Rolle, wenn es hier zu

2 US DoD (Department of Defense) (2007). Research \& Engineering Strategic Plan. Washington DC, S. 5 (Übersetzung des Autors). In http://www.dtic. mil/cgi-bin/GetTRDoc?Location=U2\&doc=GetTRDoc.pdf\&AD=ADA472100 (2.1.2013).
Begrenzungen kommen soll. Würden sie ein aufgeklärteres Konzept nationaler Sicherheit verfolgen, fiele es ihnen leichter, Beschränkungen zuzustimmen. Denn dann würden sie den Vorrang nicht so sehr auf die eigene militärische Stärke legen, sondern die internationalen Konsequenzen der Einführung neuer Waffentechniken bei vielen anderen Staaten mitbedenken und die eigene Sicherheit im internationalen Kontext eingebettet sehen. Diese Sichtweise in den USA zu stärken, ist vor allem eine Aufgabe politischer Kräfte im Land selbst, aber verbündete (und andere) Staaten können - nicht nur durch Gespräche, auch durch Begrenzungsinitiativen einen gewissen Beitrag leisten, mehr als das bisher geschieht.

In Bezug auf (neuartige) unbemannte Kampffahrzeuge (für Luft, Land, Wasser) stehen dringende Aufgaben an. Am besten wäre ein komplettes Verbot. Wenn das nicht erreichbar sein sollte, weil gegenwärtig immer mehr Staaten nach bewaffneten Drohnen streben, müsste es zumindest zu qualitativen und quantitativen Beschränkungen kommen. In Europa gelten für bewaffnete Land- und Luftfahrzeuge schon der KSE-Vertrag und das Wiener Dokument der Organisation für Sicherheit und Zusammenarbeit in Europa (OSZE). Ähnliche Regelungen sind auch in anderen Weltregionen nötig. Kleine und kleinste Fahrzeuge sollten (fast) ganz verboten werden. Verfahren zur Verifikation können sich an den eingeführten Mechanismen der Notifikation und Vor-Ort-Inspektion mit Gerät orientieren.

Wegen der Gefahren für Kriegsvölkerrecht und Stabilität sowie aus ethischen Erwägungen sollten Waffensysteme, die autonom Ziele auswählen und angreifen können, durch eine ausdrückliche neue Regel im Kriegsvölkerrecht grundsätzlich verboten werden. Da man durch einen einfachen Softwareschalter von Fernsteuerung zu selbständigem Angriff übergehen könnte, würde die Kontrolle Einsicht in die aktuellen Steuerprogramme erfordern. So aufdringliche „Verifikation im Vorhinein“ würde von den Staaten sicher abgelehnt. Aber auch andere Kriegsvölkerrechtsregeln können erst im Nachhinein überprüft werden. Hier ist eine Pflicht denkbar, die Sensor- und Kommunikationsdaten, die bei jedem Angriff in großer Menge anfallen, zu speichern und im Verdachtsfall z.B. dem Internationalen Komitee des Roten Kreuzes (IKRK) zugänglich zu machen. Das wäre zwar zunächst ein erheblicher Schritt, aber in anderen Bereichen sind vertrauliche Überprüfungen durch das IKRK akzeptiert.

Die Einhaltung der hier empfohlenen Beschränkungen ferngesteuerter bewaffneter unbemannter Fahrzeuge kann noch mit den eingeführten Verfahren überprüft werden. Das Scheitern des BWÜ-Protokolls zeigt aber schon das Problem, das entsteht, wenn die nachzuweisenden Objekte klein sind, an vielen verschiedenen Orten gehandhabt werden könnten und Inspektionen Militär oder Industrie zu aufdringlich erscheinen. Zukünftige revolutionäre Technologien werden dies erheblich verschärfen. Dazu gehören zum einen Produkte militärischer FuE, darunter kleine und kleinste Waffensysteme, vielleicht in Form von Hybridinsekten, die auch in Schwärmen eingesetzt werden könnten. Zum anderen handelt es sich um leicht verfügbare Herstellungstechniken, die aus wenigen Rohstoffen unter Softwaresteuerung „reale Dinge“ machen, wie 3-D-Drucker, die beliebige Teile aus Kunststoff, Metall und Keramik 
erzeugen, und DNS-Synthetisierer, die beliebige Genome zusammensetzen können. Solche Geräte werden immer billiger und könnten (fast) jedem die Möglichkeit geben, aus heruntergeladener Information gefährliche Hardware zu machen. Des Weiteren arbeiten Staaten geheim an Cyberangriffen. Für diese Bereiche internationale Verbote oder Begrenzungen zu vereinbaren, würde sehr aufdringliche Verifikation erfordern Inspektionen in (fast) jedem Land überall und jederzeit. Solche Inspektionsrechte bestehen innerhalb der meisten Staaten im zivilen Bereich für Gewerbeaufsicht, Polizei und Justiz. Im internationalen System müssten sie jedoch freiwillig vereinbart werden und die Streitkräfte mit umfassen - die aber Geheimhaltung benötigen, wenn sie im bewaffneten Konflikt siegen sollen.

Können sich die relevanten Staaten nicht auf Begrenzungen mit derart umfangreichen Kontrollen einigen, stellt sich die Grundsatzfrage, ob das dann zu erwartende unbegrenzte Wettrüsten und die militärische Destabilisierung das internationale System dauerhaft überfordern, oder ob die Menschheit es schaffen wird, internationale Sicherheit auf neue Art zu organisieren - mit einem Gewaltmonopol bei demokratisierten Vereinten Nationen (VN) und einer freiwillig reduzierten Souveränität der Staaten. Diese Idee ist nicht neu, man denke an die Weltföderalisten der 1930er und 1940er Jahre, den Baruch-Plan der USA zur Internationalisierung der Kernwaffen und Kontrolle der Kernenergie (1946) oder Carl Friedrich von Weizsäckers Konzept der Weltinnenpolitik (1963). Bislang von vielen als unrealistisch angesehen, könnten aus den ungeahnten Gefahren einer militärischen Nutzung revolutionärer Technologien zusätzliche Argumente für einen grundsätzlichen Politikwechsel entstehen. Gemeinsame Klimagefahren, Verflechtungen im Bereich der Ökonomie und des Internets sprechen schon heute dafür. Erste Ansätze gibt es bereits: bei den Vereinten Nationen, den internationalen Gerichtshöfen und der Europäischen Union. Sie auszubauen ist Aufgabe kluger Politik.

Dr. Jürgen Altmann, Technische Universität Dortmund, ist Physiker und Friedensforscher.

\title{
Die Rolle von kirchlichen Akteuren in Post-Konflikt-Gesellschaften
}

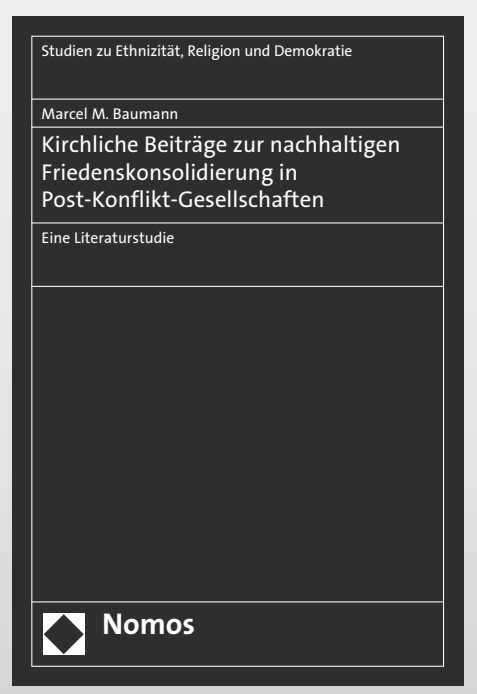

\author{
Kirchliche Beiträge zur nachhaltigen \\ Friedenskonsolidierung in Post-Konflikt-Gesellschaften \\ Eine Literaturstudie \\ Von Marcel M. Baumann \\ 2013, 134 S., brosch., 34,- - Є, ISBN 978-3-8487-0169-8 \\ (Studien zu Ethnizität, Religion und Demokratie, Bd. 15)
}

Kirchliche Akteure können in Konflikten vielfältige und sich widersprechende Rollen ausfüllen. In Fallbeispielen wie Argentinien oder Nordirland treten kirchliche Akteure als Täter auf, während im Irak oder in Nordkorea kirchliche Akteure sowie Christen als Gemeinschaft regelmäßig zu Opfern von Verfolgung und Unterdrückung werden. Kirche kann jedoch auch ein „Friedensengel“ sein, z.B. in Guatemala oder Südafrika.

Die Literaturstudie leistet eine Bestandsaufnahme über kirchliche Beiträge zur nachhaltigen Friedenskonsolidierung im globalen Konfliktgeschehen. Zum einen werden positive Beispiele für kirchliches Engagement analysiert. Zum anderen werden Fallbeispiele herausgearbeitet, in denen kirchliche Akteure als Täter auftreten und den Konflikttransformationsprozess blockieren.

Bestellen Sie jetzt telefonisch unter 07221/2104-37.

Portofreie Buch-Bestellungen unter

www.nomos-shop.de/20329

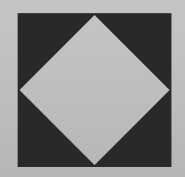

Nomos 